\title{
Elucidating the effect of intermediate resonances in the quark interaction kernel on the timelike electromagnetic pion form factor
}

\author{
Ángel S. Miramontes $\oplus^{*}$ \\ Instituto de Física y Matemáticas, Universidad Michoacana de San Nicolás de Hidalgo, \\ Morelia, Michoacán 58040, Mexico \\ Hèlios Sanchis Alepuz $\odot^{\dagger}$ \\ Silicon Austria Labs GmbH, Inffeldgasse 25F, 8010 Graz, Austria \\ Reinhard Alkofer® \\ Institute of Physics, University of Graz, NAWI Graz, Universitätsplatz, 5, 8010 Graz, Austria
}

(Received 23 March 2021; accepted 6 May 2021; published 7 June 2021)

\begin{abstract}
An exploratory study of the timelike pion electromagnetic form factor in a Poincaré-covariant bound state formalism in the isospin symmetric limit is presented. Starting from a quark interaction kernel representing gluon-intermediated interactions for valence-type quarks, nonvalence effects are included by introducing pions as explicit degrees of freedom. The two most important qualitative aspects are, in view of the presented study, the opening of the dominant $\rho$-meson decay channel and the presence of a multiparticle branch cut setting in which the two-pion threshold is crossed. Based on a recent respective computation of the quark-photon vertex, the pion electromagnetic form factor for spacelike and timelike kinematics is calculated. The obtained results for its absolute value and its phase compare favorably to the available experimental data, and they are analyzed in detail by comparing them to the expectations based on an isospin-symmetric version of a vector-meson dominance model.
\end{abstract}

DOI: 10.1103/PhysRevD.103.116006

\section{INTRODUCTION}

Hadronic timelike form factors will be measured in upcoming experiments to an unprecedented precision. An understanding of these quantities, which are displaying pronounced structures originating from hadron resonances, will contribute significantly to our knowledge on the relation between the hadrons' substructure and the hadron spectrum. On one hand, based on respective experimental and theoretical progress in the last decades, it is by now evident that hadron resonances can be, at least in principle, described in terms of quarks and gluons. The latter, being the QCD degrees of freedom, are considered to be complete in the sense that they allow for a computation of every hadronic observable. Changing the perspective in an attempt to understand the strong interaction starting from

\footnotetext{
*angel-aml@hotmail.com

helios.sanchis-alepuz@silicon-austria.com; hsanchisalepuz@ gmail

"reinhard.alkofer@uni-graz.at

Published by the American Physical Society under the terms of the Creative Commons Attribution 4.0 International license. Further distribution of this work must maintain attribution to the author(s) and the published article's title, journal citation, and DOI. Funded by SCOAP ${ }^{3}$.
}

the low-energy regime, a possible way of phrasing the phenomenon of confinement in QCD is the statement that all possible hadronic degrees of freedom also will form a complete set of physical states. Therefore, the equivalence of descriptions of observables in either quark and glue or hadronic degrees of freedom is a direct consequence of confinement and unitarity. This is the gist of a chain of arguments, which can be sophisticated and applied to many different phenomena involving hadrons. The related picture is known under the name "quark-hadron duality," and its consequences have been verified on the qualitative as well as the semiquantitative level; for a review, see, e.g., Ref. [1]. A verification of this duality is, beyond the trivial fact of the absence of colored states, the clearest experimental signature for confinement. To appreciate the scope of such a scenario, it is important to note that a perfect orthogonality of the quarkglue degrees of freedom on one hand and hadronic states on the other hand, and thus, the perfect absence of "doublecounting" in any of the two "languages," is nothing else but another way to express confinement.

Gaining insight into the interplay between formation of hadronic bound states, consisting of quarks and gluons, and the open decay channels of the respective resonance is an essential element of every study of timelike form factors in kinematic regions close to a resonance. Here, attention 
should be paid to the fact that the hadron whose form factor is investigated and the hadronic resonance that is apparent in the form factor are both to be described as composite objects of quarks and gluons. The same is true for the hadronic decay products in a hadronic or semileptonic decay of the resonance. This makes evident that an approach to calculate a timelike form factor from QCD, or from a microscopic model based on QCD degrees of freedom, faces the challenging task to treat all elements appearing in the calculation on the same footing and to a sufficient degree of sophistication if the result is intended to allow for conclusions on the dynamics underlying such a form factor.

Herein, we will report on an exploratory study of the timelike pion electromagnetic form factor using functional methods. More precisely, we will employ a combination of Bethe-Salpeter and Dyson-Schwinger equations (for recent reviews on this and related approaches, see, e.g., [2-5]). Although such an approach is capable of allowing a firstprinciple calculation (see, e.g., the computation of the glueball spectrum reported in Ref. [6]), for the task at hand, this is yet out of reach. To grasp all essential features of the pions' timelike form factor, ${ }^{1}$ one needs to describe at least (i) the pion as bound state of quark and antiquark, thereby at the same time taking into account its special role as would-be Goldstone boson of the dynamically broken chiral symmetry of QCD, (ii) the mixing, respectively, the interference of the $\rho$ meson, being described also as a quark-antiquark bound state, with a virtual photon when this photon is, in turn, coupled to a quark-antiquark pair via the fully renormalized quark-photon vertex, and (iii) the dominant decay channel of the $\rho$ meson, namely, $\rho \rightarrow \pi \pi$. The study presented here is now in two aspects exploratory. First of all, the interaction between quarks and antiquarks is modeled in such a way that the essential features, as implied by QCD and phenomenology, are taken into account but that it is still manageable in such an involved calculation. Second, in several places, we will make technical simplifications, especially when the such introduced error can, for good reasons, be assumed to be small, and the reduction in the computing time needed is substantial. We thus aim here more for an understanding of how the different features of the form factor arise from the QCD degrees of freedom than for a quantitative agreement with the experimental data.

In the chosen model for the quark-antiquark interaction, besides a gluon-mediated interaction, pions also will be included explicitly. The reason for this is as follows: If one

\footnotetext{
${ }^{1}$ There are several investigations of the spacelike pion electromagnetic form factor in the Dyson-Schwinger-Bethe-Salpeter approach; early examples include [7-9]. A calculation of this form factor spanning the entire domain of spacelike momentum transfers is described in Ref. [10]. Within the Bethe-Salpeter formalism of covariant spectator theory, the spacelike pion electromagnetic form factor has also been calculated; see, e.g., [11]
}

were able to take into account the fully renormalized quarkgluon vertex exactly within this approach, hadronic degrees of freedom will effectively emerge and thus be included in the interaction between quarks and antiquarks, and, respectively, they will back feed on the quarks' dynamics. Due to the pions' Goldstone boson nature, and especially due to the implied small pion mass, the pions are the most important low-energy degrees of freedom within the strong interaction, as, e.g., also elucidated by chiral perturbation theory. As, in order to describe the physics of decays, nonvalence effects need to be taken into account, it is some minimal requirement for the investigation reported here to include pions as the most important nonvalence-type interaction mediator in the sub-GeV region.

The interaction model herein is also chosen in view of a possible generalization to the study of baryon form factors, and hereby, especially the nucleons' timelike form factor. In this respect, one can build on existing calculations of spacelike form factors from bound state amplitudes; see, e.g., Refs. [3,12-16] for some recent respective work. A thorough understanding of the proton timelike form factor at very low $Q^{2}$ is a very timely subject as the upcoming PANDA experiment possesses the unique possibility to measure the proton's electromagnetic form factors in the so-called unphysical region through the process $\bar{p} p \rightarrow l^{+} l^{-} \pi^{0}, l=e, \mu[17]$. At large $Q^{2}$, the question of the onset of the convergence scale between the spacelike and the timelike form factors arises.

However, also, the pions' timelike electromagnetic form factor will be studied further by upcoming experiments, among other reasons, because recently, the consistency of the available datasets has been questioned [18]. Based on the long-known fact that the $\tau$ radiative decay allows one to extract the pion form factor [19] and that a very large number of $\tau$ leptons are produced at B-meson factories, further high-precision data in sub-GeV region will become available. $^{2}$

Besides earlier lattice QCD calculations of the pions' spacelike electromagnetic form factor [21-27], recently, calculations of the timelike pion form factor have become available [28-31]. These results typically show a good agreement with the experimental data. In those calculations, the extraction of the timelike form factor employs a parametrization based on the vector meson dominance (VMD) picture to determine the momentum dependence of the timelike pion form factor from the lattice data at discrete energies.

The pion electromagnetic form factor for space- and timelike momenta has also been investigated within a lightfront model in order to address the issue of nonvalence components of the pion and photon wave functions [32]. The employed relativistic approach is based on a

\footnotetext{
${ }^{2}$ In this work, we will compare to the dataset of Ref. [20] available at https://www.hepdata.net/record/ins728302.
} 
parametrization for the emission or absorption of a pion by a quark and on a microscopic VMD model for the dressed quark-photon vertex. Within our approach, the quark-pion coupling is given by the pion's Bethe-Salpeter amplitude, and the fully dressed quark-photon vertex has been calculated [33] in complete agreement with the computation of the pion electromagnetic form factor reported herein.

The exploratory calculation presented herein is done in the isospin symmetric limit. One of the effects of isospin breaking clearly visible in the timelike pion form factor is $\rho-\omega$ mixing; see, e.g., the review [34]. To this end, we will employ the VMD-based fit given in [34] and modify it such that an expected form of the pion form factor without this mixing effect is extracted. ${ }^{3}$ Furthermore, we will present a simplified but numerically quite accurate VMD parametrization of the timelike form factor, which then serves as a basis for a detailed analysis of our results. Here, the focus is more on a comparison of our results with the form expected on the basis of the VMD parametrization than on numerical agreement. ${ }^{4}$

This paper is organized as follows: In Sec. II, we review some facts about the electromagnetic pion form factor, employ the VMD-based fit given in [34] to remove the $\rho-\omega$ mixing effects, and provide an expected form of the pion form factor. In Sec. III, we present our approach based on Bethe-Salpeter and Dyson-Schwinger equations. Our results are presented and analyzed in Sec. IV. In Sec. V, we present conclusions and an outlook. Some technical details are deferred to two appendices.

\section{THE TIMELIKE ELECTROMAGNETIC PION FORM FACTOR}

The pion, being a composite object, does not have a pointlike interaction with the electromagnetic field, and the related substructure, the pion being a pseudoscalar, is related to one form factor. Considering, for example, the scattering of an electron off a pion $\pi^{+}$, one can describe the leptonic part of the interaction quite precisely in lowestorder perturbation theory; i.e., one considers the process in which the electron emits a virtual photon, and the latter couples to the pion. Defining the form factor $F_{\pi}(s)$ via the relation,

$$
\left\langle\pi^{+}\left(p_{1}\right)\left|j_{\text {e.m. }}^{\mu}\right| \pi^{+}\left(p_{2}\right)\right\rangle=e\left(p_{1}+p_{2}\right)^{\mu} F_{\pi}\left(q^{2}\right),
$$

where $q^{\mu}=p_{1}^{\mu}-p_{2}^{\mu}$ is the virtual photon momentum, and $e$ is the elementary electric charge. The $S$-matrix element for

\footnotetext{
${ }^{3}$ In Ref. [35], another method has been used to remove the $\rho-\omega$ mixing effects from the data.

${ }^{4}$ A precise representation of the timelike form factor requires parametrizations, including excited $\rho$ mesons; respective examples can be found in Refs. [36,37]. Two-photon effects, on the other hand, can very likely be safely neglected; for a corresponding study of the form factor at large momentum transfer, see [38].
}

electron-pion scattering is then proportional to the form factor,

$$
\begin{aligned}
i \mathcal{M}_{e \pi \rightarrow e \pi}= & e\left(p_{1}+p_{2}\right)^{\mu} F_{\pi}\left(q^{2}\right) D_{\text {photon }}^{\mu \nu}(q) \\
& \left(i e \bar{u}\left(k_{1}, s_{1}\right) \gamma_{\nu} u\left(k_{2}, s_{2}\right)\right),
\end{aligned}
$$

where $D_{\text {photon }}^{\mu \nu}(q)$ is the photon propagator, and $u\left(k_{i}, s_{i}\right)$ is the electron spinor; see, e.g., Sec. 8.4 of [39] for more details. The kinematics of this scattering process is such that the photon momentum is spacelike, and without loss of generality, one can assume the form factor $F_{\pi}(s)$ to be real. Charge conservation requires that for a real photon, one has $F_{\pi}(0)=1$.

Turning to the process of electron-positron annihilation into a pion pair, the corresponding $S$-matrix element is again proportional to the form factor (see, e.g., Sec. 8.5 of [39]),

$$
\begin{aligned}
i \mathcal{M}_{e^{+} e^{-} \rightarrow \pi^{+} \pi^{-}}= & e\left(p_{1}+p_{2}\right)^{\mu} F_{\pi}\left(q^{2}\right) D_{\text {photon }}^{\mu \nu}(q) \\
& \left(-i e \bar{v}\left(k_{1}, s_{1}\right) \gamma_{\nu} u\left(k_{2}, s_{2}\right)\right)
\end{aligned}
$$

with appropriately redefined momenta. Especially, the virtual photon momentum is now timelike (cf., Fig. 1), and one measures in such an annihilation process to a pion pair the form factor for timelike momenta. Above the two-pion production threshold, i.e., in the physical region, the corresponding cut in the amplitude (3) necessitates one to treat the timelike pion form factor as a complex quantity, and it fulfills the dispersion relation:

$$
F_{\pi}\left(q^{2}\right)=1+\frac{q^{2}}{\pi} \int_{4 m_{\pi}^{2}} d s \frac{\mathcal{I} m F_{\pi}(s)}{s\left(s-q^{2}-i \epsilon\right)} .
$$

Especially, it is expected that the phase of the pion form factor varies strongly in a two-pion resonance region. Below the inelastic threshold, i.e., for $s<4 m_{\pi}^{2}$, the timelike pion form factor is, via Watson's final state theorem, related to the isovector P-wave scattering phase shift $\delta_{1,1}(s)$ :

$$
\begin{aligned}
\operatorname{Im}_{\pi}\left(q^{2}\right) & =\frac{1}{2 i}\left(1-e^{-2 i \delta_{1,1}(s)}\right) F_{\pi}(s+i \epsilon) \\
& =\sin \left(\delta_{1,1}(s)\right) e^{-i \delta_{1,1}(s)} F_{\pi}(s+i \epsilon) .
\end{aligned}
$$

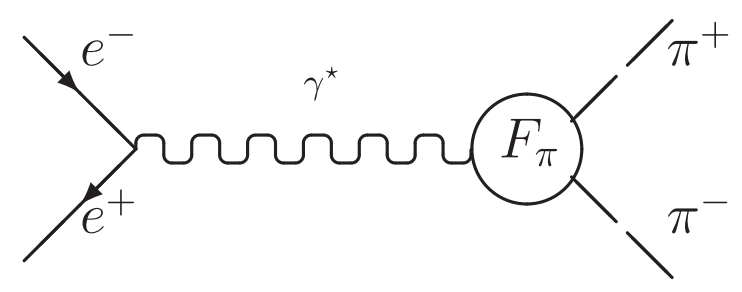

FIG. 1. Electron-positron pair annihilating to a virtual photon with timelike momentum, which then decays to a pion pair. 
As depicted in Fig. 1, the pion form factor contains, for timelike as well as for spacelike photon virtualities, all kind of interaction processes turning a photon into a pion pair. As strong interaction processes dominate the corresponding amplitude, and as gluons do not couple directly to photons, one decisive element of the pion form factor is the amplitude describing how the photon couples to a quark, taking hereby all possible contributing QCD processes into account. This amplitude is exactly the full quark-photon vertex. As we will see in the following, this correlation function carries information about the virtual photon's hadronic substructure. And, as will be described in detail in the next section, the other quantities needed then for calculating the pion form factor are the fully renormalized quark propagator and the pion bound state amplitude, the latter describing how an antiquark and a quark form a pionic bound state.

While our calculation is based on QCD degrees of freedom, it is capable of providing an understanding why in the resonance region, a vector-meson dominance (VMD) picture provides very good results for the timelike pion form factor. In addition, it will elucidate to which extent a VMD picture might be applicable in other kinematic regions.

In the VMD picture, the hadronic contribution to the photon propagator is given by mixing with electrically neutral vector mesons. Restricting to light-quark mesons, the corresponding vector meson is the $\rho^{0}$, i.e., the uncharged member of the isotriplet of vector mesons. In a would-be isospin symmetric world, this would be the only vector meson below one $\mathrm{GeV}$ with which a virtual photon mixes because the isosinglet $\omega$ will not mix with the photon due to $G$ parity.

In the real world, isospin symmetry is broken, and one of the many effects of isospin breaking is $\rho-\omega$ mixing; see, e.g., the review [34]. This mixing and the resulting interference of states lead to quite some pronounced structure in the timelike pion form factor around $m_{\omega}^{2}$. Indeed, it is by now well understood that the sharp dip in the experimental data stems from a combination of $\rho-\omega$ mixing and interference effects between the decays $\rho \rightarrow \pi \pi$ and $\omega \rightarrow \pi \pi$, the latter being isospin breaking; see, e.g., Ref. [34]. Moreover, it has been estimated that this combination of mixing and interference effects decreases the height of the bump of the order of up to 10\% (see [35] and references therein); cf., also Fig. 4 of [40] in which a calculation of the the timelike pion form factor employing a dispersion representation of meson loop diagrams has been reported.

As the here presented exploratory calculation is performed in the isospin limit, ${ }^{5}$ and thus, $\rho-\omega$ mixing is

\footnotetext{
${ }^{5}$ Isospin breaking and thus, the effect of $\rho-\omega$ mixing on the pion form factor is currently under investigation; the corresponding results will be published elsewhere.
}

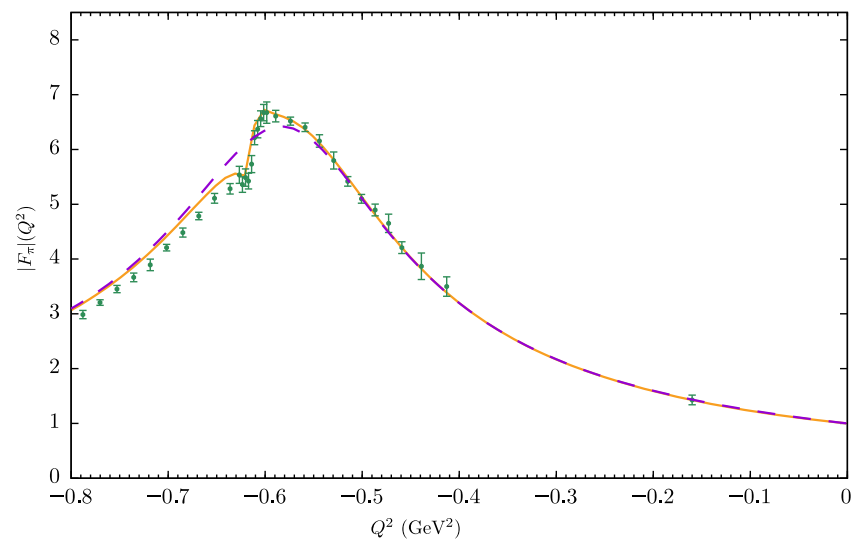

FIG. 2. Absolute value of the pion form factor in the timelike $\left(Q^{2}<0\right)$ domain from the VMD-based fit given in Ref. [34] (full line) in comparison to the experimental data [20]. The dashed line is based on the same expression but the mixing matrix element put to zero, $\Pi_{\rho \omega}=0$.

neglected, we estimate its effect by comparing the VMDbased fit to the pion form factor given in Ref. [34] with a plot of the same expression but the mixing matrix element put to zero, $\Pi_{\rho \omega}=0$ (see Fig. 2). As expected, the resulting curve is much smoother than the one including the $\rho-\omega$ interference effect. Around the $\omega$ mass, the deviation of the two curves can be as large as almost 10\%; however, this effect is limited to a small interval, and the two curves are practically indistinguishable outside this small interval. Therefore, we expect our calculation to reproduce all qualitative features of the curve representing the case with $\rho-\omega$ mixing switched off and to be in a reasonable quantitative agreement with it.

It is instructive to analyze the momentum behavior of a simplified version of the fit given in Ref. [34]. In order to be in agreement with the notation, in the following presentation, we introduce the photon virtuality with the convention that $Q^{2}<0$ corresponds to the timelike region. In the above used fit, a momentum dependent expression for the width of the $\rho$ meson is used. It displays the two-pion cut,

$$
\Gamma_{\rho}\left(Q^{2}\right) \propto\left(-Q^{2}-4 m_{\pi}^{2}\right)^{3 / 2} \Theta\left(-Q^{2}-4 m_{\pi}^{2}\right)
$$

which is important for a qualitatively correct analytic structure of the pion form factor. However, especially close to the maximum of the pion form factor, the pion mass is quantitatively negligible, and for vanishing pion mass, the momentum dependent width assumes for timelike $Q^{2}<0$ the relatively simple form,

$$
\Gamma_{\rho}\left(Q^{2}\right)=\bar{\Gamma}_{\rho}\left|Q^{2}\right| / m_{\rho}^{2},
$$


where $\bar{\Gamma}_{\rho}=\Gamma_{\rho}\left(-m_{\rho}^{2}\right)$. This then leads to the simplified but still quite accurate form for the fit, ${ }^{6}$

$$
\begin{aligned}
F_{\pi}\left(Q^{2}+i \epsilon\right)= & 1-\frac{g_{\rho \pi \pi}}{g_{\rho}} \frac{Q^{2}\left(Q^{2}+m_{\rho}^{2}\right)}{\left(Q^{2}+m_{\rho}^{2}\right)^{2}+Q^{4} \bar{\Gamma}^{2} / m_{\rho}^{2}} \\
& +i \frac{g_{\rho \pi \pi}}{g_{\rho}} \frac{Q^{4} \bar{\Gamma} / m_{\rho}}{\left(Q^{2}+m_{\rho}^{2}\right)^{2}+Q^{4} \bar{\Gamma}^{2} / m_{\rho}^{2}}
\end{aligned}
$$

where $\epsilon \rightarrow 0^{+}$has been introduced to fix the sign of the imaginary part. Hereby, the coupling constant $g_{\rho \pi \pi}$ is determined from $\Gamma_{\rho \pi \pi}=\bar{\Gamma}=149 \mathrm{MeV}$ to be $g_{\rho \pi \pi} \approx 6$, and $g_{\rho}$ is a parameter reflecting the strength of the $\gamma-\rho^{0}$-mixing, which is then described by the effective Lagrangian,

$$
\mathcal{L}_{\rho \gamma}=-\frac{e m_{\rho}^{2}}{g_{\rho}} \rho_{\mu}^{0} A^{\mu}
$$

From the partial width $\Gamma_{\rho e^{+} e^{-}}=7 \mathrm{keV}$, one infers $g_{\rho} \approx 5$.

The form given in Eq. (8) motivates one to compare the obtained results for the real and the imaginary part of the timelike form factor from our calculation to a rational, also known as, Padé fit. Phrased otherwise, Eq. (8) formalizes the expectation for the timelike form factor based on the VMD picture, and our results based on the quark-photon vertex function and the pion bound state amplitude will be analyzed by discussing how much they extend beyond to this form.

\section{DYSON-SCHWINGER AND BETHE-SALPETER FORMALISM}

We determine the necessary input for the calculation of the pion electromagnetic form factor using a combination of Bethe-Salpeter (BSE) and Dyson-Schwinger equations (DSE). To make this presentation self-contained, we summarize in this section the most relevant aspects of the approach; for more details see, e.g., the recent reviews [2-5] as well as references therein. All expressions in the following are understood to be formulated in Euclidean momentum space, i.e., after a Wick rotation.

In the DSE and BSE formalism, the fully dressed quarkphoton vertex $\Gamma^{\mu}$, which describes the interaction between quarks and photons in a quantum field theory, can be obtained as the solution of an inhomogeneous BSE,

\footnotetext{
${ }^{6}$ In contrast to a constant width approximation, the pole is in this parameterization and thus, in the employed fit not located at $Q^{2}=-m_{\rho}^{2}+i m_{\rho} \bar{\Gamma}_{\rho}$ but at $Q^{2}=\left(-m_{\rho}^{2}+i m_{\rho} \bar{\Gamma}_{\rho}\right) /\left(1+\bar{\Gamma}_{\rho}^{2} / m_{\rho}^{2}\right)$; i.e., the real and imaginary part of the pole position are decreased by $3.7 \%$ if the same values for the $\rho$ 's mass and width are used.
}

$$
\begin{aligned}
\left(\Gamma^{\mu}\right)_{a \alpha, b \beta}(p, Q)= & Z_{2}\left(\gamma^{\mu}\right)_{a b} t_{\alpha \beta} \\
& +\int_{q} K_{a \alpha, b \beta}^{r \rho, s \sigma}(Q, p, q) S_{r \rho, e \epsilon}\left(k_{1}\right) \\
& \times\left(\Gamma^{i, \mu}\right)_{e \epsilon, n \nu}(Q, q) S_{n \nu, s \sigma}\left(k_{2}\right) .
\end{aligned}
$$

Here, $Q$ is the photon momentum, $p$ is the relative momentum between quark and antiquark, $q$ is an internal relative momentum that is integrated over, and the internal quark and antiquark momenta are defined as $k_{1}=q+Q / 2$ and $k_{2}=q-Q / 2$, respectively, such that $Q=k_{1}-k_{2}$ and $q=\left(k_{1}+k_{2}\right) / 2$. Latin letters represent Dirac indices, and Greek letters represent flavor indices. The isospin structure of the vertex is given by $t_{\alpha \beta}=\operatorname{diag}(2 / 3,-1 / 3)$. The Dirac structure of the vertex can be expanded in a basis consisting of 12 elements [33], and all of them are considered in our calculation.

Similarly, mesons as bound states of two quarks are described in this framework by Bethe-Salpeter amplitudes $\Gamma$, which are obtained as solutions of a homogeneous BSE,

$$
\begin{aligned}
(\Gamma)_{a \alpha, b \beta}(p, P)= & \int_{q} K_{a \alpha, b \beta}^{r \rho, s \sigma}(P, p, q) \\
& \times S_{r \rho, e \varepsilon}\left(k_{1}\right)(\Gamma)_{e \varepsilon, n \nu}(q, P) S_{n \nu, s \sigma}\left(k_{2}\right),
\end{aligned}
$$

where, for clarity, we have here used $P$ for the total meson momentum (instead of $Q$ as above). For pions, the Dirac part of the Bethe-Salpeter amplitude $\Gamma$ can be expanded in a tensorial basis with four elements.

In the equations above, the interaction kernel $K$ describes the interaction between quark and antiquark, and $S$ is the fully dressed quark propagator. We will discuss in detail the interaction kernels below. The quark propagator $S(p)$ is obtained as the solution of the quark DSE,

$$
S^{-1}=S_{0}^{-1}-Z_{1 f} \int_{q} \gamma_{\mu} S(q) \Gamma_{\nu}^{q g l}(q, k) D_{\mu \nu}(k)
$$

with $S_{0}^{-1}$ the renormalized bare propagator,

$$
S_{0}^{-1}(p)=Z_{2}\left(i \not p+Z_{m} m\right)
$$

and $Z_{1 f}, Z_{2}$, and $Z_{m}$ are renormalization constants, $m$ is the (renormalization-point dependent) current quark mass, $\Gamma^{q g l}$ is the full quark-gluon vertex, and $D_{\mu \nu}$ is the full gluon propagator which, in the Landau gauge, is parametrized as

$$
D_{\mu \nu}(k)=\left(\delta_{\mu \nu}-\frac{k_{\mu} k_{\nu}}{k^{2}}\right) \frac{Z\left(k^{2}\right)}{k^{2}},
$$

with $Z\left(p^{2}\right)$ being the gluon dressing function. For simplicity, we have suppressed the color indices. 


\section{A. Interaction kernels}

The interaction kernel $K$ in Eqs. (9) and III encodes all possible interactions processes between a quark and an antiquark. In a diagrammatic representation, it contains a sum of infinitely many terms. In practical calculations, the expansion of the interaction kernel must be truncated to a sum of a finite number of terms, chosen such that the relevant dynamics and global symmetries are correctly implemented. Chiral symmetry and its dynamical breaking ensures that pions are massless bound states in the chiral limit as a consequence of Goldstone's theorem. On the other hand, U(1) vector symmetry ensures charge conservation in electromagnetic processes. Chiral symmetry will be correctly implemented in the DSE and BSE formalism only if the kernel fulfills the axial-vector Ward-Takahashi identity (Ax-WTI),

$$
\begin{aligned}
& i \Sigma_{a r}\left(p_{+}\right) \gamma_{r b}^{5} t_{\alpha \beta}^{i}+i \gamma_{a r}^{5} \Sigma_{r b}\left(p_{-}\right) t_{\alpha \beta}^{i} \\
& =\int_{q} K_{a \alpha, b \beta}^{r \rho, s \sigma}(Q, p, q)\left[i t_{\rho \nu}^{i} \gamma_{r n}^{5} S_{n \nu, s \sigma}\left(q_{-}\right)+i S_{r \rho, e \varepsilon}\left(q_{+}\right) \gamma_{e s}^{5} t_{\varepsilon \sigma}^{i}\right]
\end{aligned}
$$

with $\Sigma$ the quark self-energy and $v_{ \pm}=v \pm Q / 2$. Similarly, vector symmetry will be correctly implemented if the kernel satisfies the vector Ward-Takahashi identity (V-WTI),

$$
\begin{aligned}
& i \Sigma_{a b}\left(p_{+}\right) t_{\alpha \beta}^{i}+i \Sigma_{a b}\left(p_{-}\right) t_{\alpha \beta}^{i} \\
& \quad=\int_{q} K_{a \alpha, b \beta}^{r \rho, s \sigma}(Q, p, q)\left[i t_{\rho \nu}^{i} S_{r \nu, s \sigma}\left(q_{-}\right)-i S_{r \rho, s \varepsilon}\left(q_{+}\right) t_{\varepsilon \sigma}^{i}\right],
\end{aligned}
$$

In DSE and BSE studies, the most widely used truncation is the so-called rainbow-ladder (RL) truncation, whereby the BSE kernel consists of a vector-vector gluon exchange, namely (omitting again color indices),

$$
K_{a \alpha, b \beta}^{r \rho, s \sigma}(Q, p, q)=\alpha\left(k^{2}\right) \gamma_{a r}^{\mu} \gamma_{s b}^{\nu} D^{\mu \nu}(k) \delta^{\alpha \rho} \delta^{\sigma \beta},
$$

with $k=p-q$ the gluon momentum. In order to preseve the Ax-WTI and V-WTI, the kernel (16) is used in combination with a truncated quark DSE, defined by the replacement,

$$
Z_{1 f} \gamma_{\mu} Z\left(k^{2}\right) \Gamma_{\nu}^{\mathrm{qgl}}(q, p) \rightarrow Z_{2}^{2} \gamma_{\mu} 4 \pi \alpha\left(k^{2}\right) \gamma_{\nu}
$$

such that $\alpha\left(k^{2}\right)$ provides an effective coupling that describes the strength of the quark-antiquark interaction. To parametrize this effective interaction, we use the MarisTandy model $[41,42]$, $\alpha\left(q^{2}\right)=\pi \eta^{7}\left(\frac{q^{2}}{\Lambda^{2}}\right)^{2} e^{-\eta^{2} \frac{q^{2}}{\Lambda^{2}}}+\frac{2 \pi \gamma_{m}\left(1-e^{-q^{2} / \Lambda_{t}^{2}}\right)}{\ln \left[e^{2}-1+\left(1+q^{2} / \Lambda_{\mathrm{QCD}}^{2}\right)^{2}\right]}$,

where the second term on the right-hand side reproduces the one-loop QCD behavior of the quark propagator in the ultraviolet, and the Gaussian term provides enough interaction strength for dynamical chiral symmetry breaking to take place. The model parameters $\Lambda$ and $\eta$ are determined as explained in the next section. The scale $\Lambda_{t}=1 \mathrm{GeV}$ is introduced for technical reasons and has no impact on the results. For the anomalous dimension, we use $\gamma_{m}=12 /\left(11 N_{C}-2 N_{f}\right)=12 / 25$, with $N_{f}=4$ flavors and $N_{c}=3$ colors. For the QCD scale, we use $\Lambda_{\mathrm{QCD}}=$ $0.234 \mathrm{GeV}$.

In the RL truncation, bound states cannot develop a decay width; correspondingly, their masses are real numbers. Note that bound states, determined as solutions of BSEs, appear as poles in Green's functions with the corresponding quantum numbers. In the RL approximation, these poles occur for real (and in the convention employed here, negative) momentum-squared values in certain kinematic configurations. In particular, for the photon being described by a vector field, electrically neutral vector mesons appear as poles of the quark-photon vertex. In the RL approximation, these poles are located at negative and real values of $Q^{2}$ (for which $M^{2}=-Q^{2}$, with $M$ being the mass of the vector meson). Such poles in the quarkphoton vertex also manifest as poles in the calculation of timelike form factors, in contradiction with phenomenology. Generally speaking, any physical phenomenon that is triggered by the presence of virtual intermediate particles, as, e.g., decays, will be absent from any calculation using the RL truncation only.

It is possible to improve the RL truncation in this respect by reintroducing ${ }^{7}$ the presence of intermediate particles explicitly. The simplest implementation of such an idea was introduced in $[43,44],{ }^{8}$ where, based on the role of the pion as lightest hadron, explicit pion-quark interactions were introduced in the truncated quark DSE and in the BSE kernel $K$, with the pion-quark interaction vertex given by the pion Bethe-Salpeter amplitude $\Gamma$, calculated consistently via a truncated BSE. The corresponding additional BSE kernels are given in Appendix A and shown in Fig. 8, and the technical difficulties arising for timelike momenta when those kernels are used in BSEs have been described

\footnotetext{
${ }^{7}$ Note that effects of intermediate virtual states like the decay of hadrons are, in principle, present in the full quark-gluon vertex which, however, is drastically simplified in the RL truncation.

${ }^{8}$ See, however, Ref. [45] for considering pion loop contributions to the electromagnetic pion radius in the DSE and BSE approach. An alternative way is to introduce two-pion states via an explicit two-pion component in the bound state amplitude; see Ref. [46] and references therein.
} 


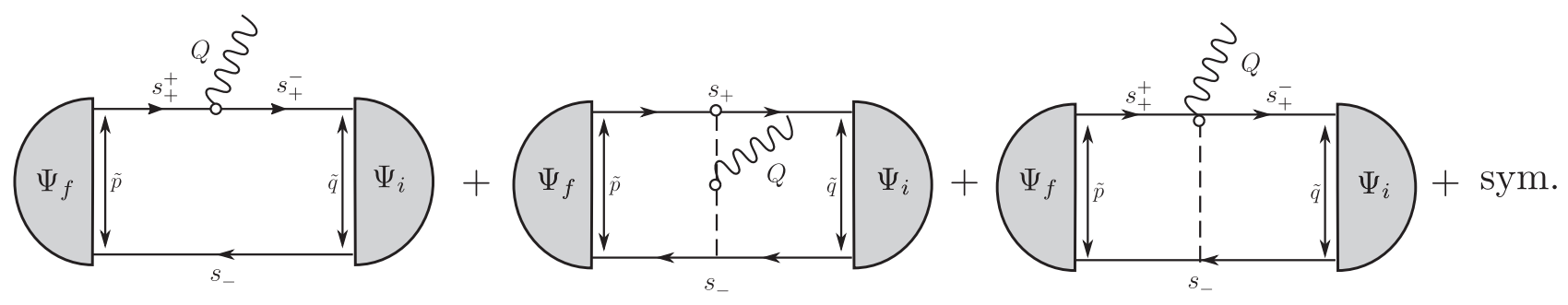

FIG. 3. Diagrams relevant for the calculation of the pion form factor in the truncation employed herein, as determined by the gauging method. Solid lines represent propagating quarks and dashed lines propagating pions. Wavy lines correspond to the external photon. The impulse approximation implies considering the first diagram and the corresponding permutation only.

in detail in [33]. These type of kernels enable the possibility of intermediate virtual decays in the BSE interaction kernel to occur. As a consequence, certain BSE solutions signal a finite decay width and thus, represent (a) hadron resonance (s). For example, the description of the $\rho$ meson as a finitewidth resonance is then mostly due to the intermediate process $\rho \rightarrow \pi \pi$ (see [47] for a treatment in the here discussed approach as well as [48,49] and references therein for respective calculations based on DSEs and BSEs) and the partial decay width to the latter process representing more than $99 \%$ of the total $\rho$ decay width. Additionally, and thereby completing the physical effects of intermediate virtual $\pi \pi$-states, in this truncation, the quark-photon vertex develops a multiparticle branch cut along the negative real $Q^{2}$ axis, starting as expected at the two-pion production threshold [33]. Clearly, including these two effects of the intermediate virtual $\pi \pi$-states is especially important when it comes to the calculation of the pion form factor for timelike momenta in the sub-GeV kinematic region.

We wish to stress here that, for computational feasibility, for the pion vertices in (A1)-(A4) we used the leading $\gamma_{5}$ component of the pion Bethe-Salpeter amplitude in the chiral limit, given by $B / f_{\pi}$, with $B$ one of the quark's dressing functions [see Eq. (A5)]. On the other hand, the pion amplitudes used in the form factor calculation of Eq. (19) are considered in full, including their leading and subleading contributions. In that regard, our calculations contain two types of treatments of pions.

Even though the "pionic" kernels (A1)-(A4) are phenomenologically justified and, as we will see in the next section, constitute a first step in the correct direction, it must be noted that they have not been (yet) rigorously derived from QCD. Lacking a solid quantum-field theoretical basis, the use of these kernels (A1)-(A4) comes with some shortcomings, especially as it implies that the AxWTI and V-WTI are not fulfilled simultaneously. Briefly, the origin of the problem is that, microscopically, the quark-pion interaction used in this paper represents only a partial resummation of diagrams involving quarks and gluons, as they appear in the quark-gluon vertex [43]. These diagrams, however, would not necessarily manifest in the same way in the Bethe-Salpeter kernel and, thus, their resummation in pionic degrees of freedom must be adjusted in order to keep consistency between DSEs and BSEs and preserve the relevant symmetries. Indeed, one can choose to preserve either the Ax-WTI (and hence, chiral symmetry) or the V-WTI (and hence, charge conservation), but not both $[33,43]$. It turns out, however, that the respective violation of either of them induce typically only small errors in physical observables, as we will demonstrate for some quantities in the next section.

\section{B. Form factor calculation}

Meson form factors are extracted from a current $J^{\mu}$ encoding the coupling of a meson to an external electromagnetic current. In the BSE framework, the current is calculated by means of the coupling of an external photon to each of the constituents of the bound state, as specified by a procedure known as gauging and developed in [5054]. The conserved current $J^{\mu}$ that describes the coupling of a single photon with a quark-antiquark, a three-quark or other multiquark system is given by

$$
J^{\mu}=\bar{\Psi}_{f} G_{0}\left(\Gamma^{\mu}-K^{\mu}\right) G_{0} \Psi_{i},
$$

with $\Psi_{i, f}$ the incoming and outgoing Bethe-Salpeter amplitudes of the meson, the baryon, or some other multi-quark state, and $G_{0}$ represents the appropriate product of dressed quark propagators. This equation is shown diagrammatically for the quark-antiquark-meson case in Fig. 3. The term $\Gamma^{\mu}$ represents the impulse approximation diagrams where the photon couples to the valence quarks only,

$$
\Gamma^{\mu}=\left(S^{-1} \otimes S^{-1}\right)^{\mu}=\Gamma^{\mu} \otimes S^{-1}+S^{-1} \otimes \Gamma^{\mu},
$$

with $\Gamma^{\mu}$ the quark-photon vertex. The term $K^{\mu}$ describes the interaction of the photon with the Bethe-Salpeter kernel, which, in our truncation, includes the coupling of the photon to the quark-pion vertex and to the propagating pions (second and third diagram in Fig. 3). Including both terms in (19) is necessary in order to implement current conservation precisely. Note that the s- and u-channel pion decay terms (most right diagram in Fig. 8) do not contribute to the term $K^{\mu}$, nor via seagulls, the reason being that trying 
to include them would leave a $\pi \pi \pi$ amplitude on one side of the respective full diagram. However, the new vertices appearing in the term $K^{\mu}$, the coupling of the photon to the quark-pion vertex and to the propagating pions, represent an enormous computational challenge. Given the exploratory purpose of the present calculation, we thus decided to omit the coupling of the photon to the quark-pion vertex and to the propagating pions and to consider the impulse diagram only (first diagram in Fig. 3). As we will show in the next section, the thereby implied violation of charge conservation is at the level of approximately one percent.

\section{RESULTS}

Following the formalism sketched above, we have calculated the pion electromagnetic form factor in the timelike $Q^{2}<0$ domain. For comparison with previous calculations, we will also show results in the spacelike $Q^{2}>0$ domain.

In our discussion in the preceding section, it remained to be explained how the parameters $\eta$ and $\Lambda$ of our interaction model (18), as well as the value of the current quark mass, are fixed. It is customary in studies using the RL truncation to adjust those parameters such that the pion decay constant agrees with the experimental value. ${ }^{9}$ In Ref. [33], the quarkphoton vertex has been calculated for the first time with the above discussed interaction kernels taken into account and also used herein. The parameters, including the isospin symmetric light-quark current mass $m_{q}$, were adjusted such that the pion mass and decay constant as well as the $\rho$ meson mass have been correctly reproduced in the employed approximation. Here, only the gluon- and pion-exchange kernels need to be used for fixing the parameters because the pion decay kernels (A3) and (A4) do not contribute to the pion BSE.

For the present exploratory calculation, we choose to adjust the parameters in a slightly different and simpler manner, especially as we aim at a qualitative understanding of the physical mechanisms involved in determining the shape of the pion form factor and not so much at achieving an accurate quantitative agreement with experiment. First, we set initially $\eta=1.5$. Second, although we assume (confirmed by our calculation) that the pion-decay kernels will not only move the $\rho$-meson pole into the complex plane but also shift down its real value, we nevertheless adjust the parameter $\Lambda$ such that we obtain a $\rho$-meson mass close to the phenomenological value already in the calculations with gluon- and pion-exchange kernels only. Third, we require it to reproduce a reasonably accurate value for the pion decay constant, namely $f_{\pi}=138 \mathrm{MeV}$. Although this value is a few percent larger than the experimental one (cf., the most recent Particle Data Group (PDG) value

\footnotetext{
${ }^{9}$ Note that, for this observable, the result is quite independent of the value of $\eta$ around $\eta=1.8$, and thus, effectively, only the parameter $\Lambda$ has to be adjusted.
}

TABLE I. The pion mass $m_{\pi}$, the pion decay constant $f_{\pi}$, and the $\rho$-meson and $\omega$-meson masses $m_{\rho}$ and $m_{\omega}$ for the two different parameterizations of the model used herein and for the case with rainbow-ladder and pion-exchange kernels but without decay kernels are shown. The light-quark mass has been set to $m_{q}=0.0068 \mathrm{GeV}$. The rightmost column displays the extracted $\rho$-meson pole position defined as $M_{\text {pole }}^{2}=M_{\rho}^{2}-i M_{\rho} \Gamma_{\rho}$, as discussed in the text. All values for dimensionful quantities are given in $\mathrm{GeV}$.

\begin{tabular}{lcccccc}
\hline \hline$\Lambda=0.78$ & $m_{\pi}$ & $f_{\pi}$ & $m_{\rho}$ & $m_{\omega}$ & $M_{\rho}$ & $\Gamma_{\rho}$ \\
\hline$\eta=1.5$ & 0.139 & 0.138 & 0.768 & 0.778 & 0.750 & 0.100 \\
$\eta=1.6$ & 0.126 & 0.138 & 0.774 & 0.784 & 0.759 & 0.105 \\
\hline \hline
\end{tabular}

$f_{\pi}^{\mathrm{PDG}}=130.50(1)(3)(13) \mathrm{MeV}$ [55]), we have adopted it in view of a compromise in the effort needed for the parameter determination and the related accuracy. Finally, we adjust $m_{q}$ to obtain the correct value for the pion mass as well. In this way, we chose the model parameters to be $\eta=1.5, \Lambda=0.78 \mathrm{GeV}$, and $m_{q}=6.8 \mathrm{MeV}$ at a renormalization scale $\mu=19 \mathrm{GeV}$. As a rudimentary test of model dependence, we additionally perform the calculations for $\eta=1.6$ as well (keeping $\Lambda$ and $m_{q}$ unchanged). The results for the pion mass and decay constant as well as for the $\rho$ meson and $\omega$-meson masses, without the pion decay kernels being taken into account, are shown in Table I.

As mentioned in the previous section, the full QCD quark-photon vertex possesses poles reflecting the masses and widths of the electrically neutral vector meson resonances. Correspondingly, and as discussed in detail in Refs. [33,47], a solution for the quark-photon vertex in the DSE and BSE framework allows one to extract the $\rho$-meson mass and width via the position of the poles of the vertex dressing functions. For the RL truncation, as well as for the RL plus pion exchange approximation, this pole is located on the real negative $Q^{2}$ axis, indicating that the $\rho$ mesons were stable for those truncations. Including the $s$ - and $u$ channel decay kernels (A3) and (A4), the pole of the dressing functions moves into the complex plane and can be extracted from the data on the real axis via a Padé fit. Parametrizing the pole position as $M_{\text {pole }}^{2}=M_{\rho}^{2}-i M_{\rho} \Gamma_{\rho}$, we extract the corresponding results for the $\rho$-meson mass and width, $M_{\rho}$ and $\Gamma_{\rho}$, for this truncation (see Table I) in reasonable agreement with the experimental values. Hereby, it has to be noted that underestimating the $\rho$ meson width does not come unexpected because taking into account only the leading $\gamma_{5}$ component of the pion BetheSalpeter amplitude in the kernels (A1)-(A4) misses some strengths therein. Whether considering in addition that the subleading pion amplitudes will provide a much better result for the $\rho$-meson width can only be answered by performing the corresponding calculation. This computation is then, however, an order of magnitude more expensive than the present exploratory calculation. 


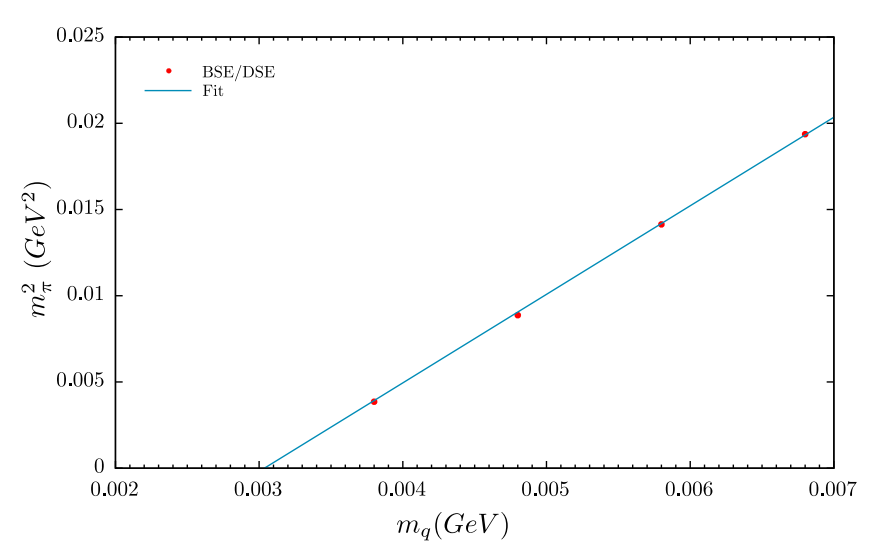

FIG. 4. The pion mass squared $m_{\pi}^{2}$ versus the current mass $m_{q}(\mu=19 \mathrm{GeV})$ for the employed truncation.

It is interesting to note that the pion exchange kernels lift the degeneracy in between the isovector $\rho$ - and the isosinglet $\omega$-meson present at the level of RL calculations. Whereas the interaction kernels in the RL truncation are, in contradiction to phenomenology, flavor blind and thus flavor $\mathrm{U}(2)$ [resp., flavor $\mathrm{U}\left(N_{f}\right)$ ] symmetric, the pion exchange kernels are not; they lead to a splitting such that $m_{\omega}-m_{\rho}=10 \mathrm{MeV}$, which compares favorably with the experimental splitting of 7-8 MeV.

We turn now to the calculation of the pion form factor.

As already indicated in the previous section, there are, besides restricting to the leading pion amplitude in the kernels (A1)-(A4), two major approximations that we must perform in order to keep the calculation technically manageable. First, using the decay kernels as described in this work entails that one must choose whether the axialvector or the vector WTI are preserved, while the other one is violated. Following [33], we choose to preserve the vector identity. A violation of the axial-vector WTI is manifested, among others, in the pion not being massless in the chiral limit, and therefore, the value of the current mass for which the pion becomes massless allows for a quantification of the violation of the axial-vector WTI. In Fig. 4, we therefore show the evolution of the pion mass with varying $m_{q}$ in the employed truncation. First, the relation is linear as expected from the Gell-Mann-Oakes-Renner relation, which is a direct consequence of the dynamical breaking of chiral symmetry. Second, the pion does not become massless in the chiral limit but for a value of the current mass $m_{q}^{(0)}(\mu=19 \mathrm{GeV})=3 \mathrm{MeV}$. On one hand, this explains the relatively large value of $m_{q}(\mu=$ $19 \mathrm{GeV})=6.8 \mathrm{MeV}$ we needed to obtain the correct pion mass: The related explicitly chiral-symmetry-breaking term is $m_{q}-m_{q}^{(0)}=3.8 \mathrm{MeV}$ and thus, much closer to what is expected from the known parameters of QCD. Second, as the masses of the vector mesons depend linearly on the current mass, the induced error on $m_{\rho}$ and $m_{\omega}$ is of the order of $m_{q}^{(0)}=3 \mathrm{MeV}$, and thus, it is as small or even smaller

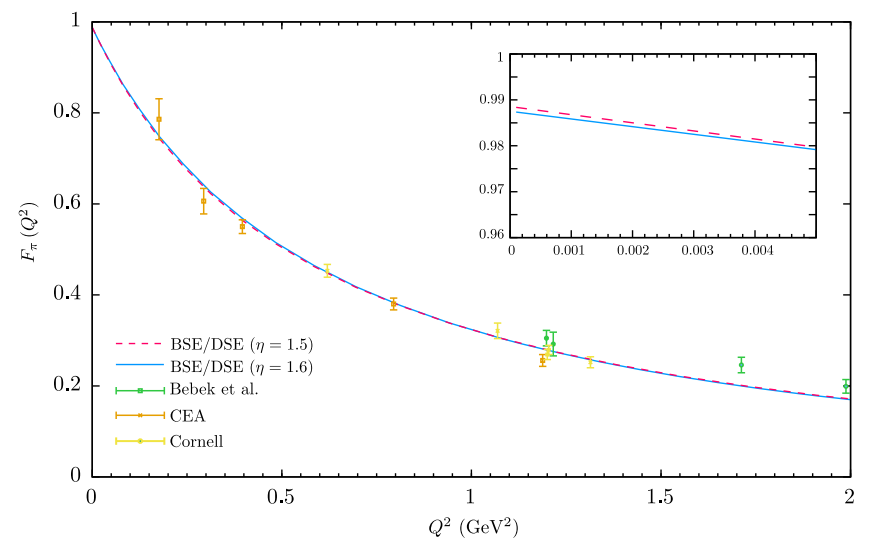

FIG. 5. Pion form factor in the spacelike $Q^{2}>0$ domain for the model parameters $\eta=1.5$ and $\eta=1.6$, as described in the text. The experimental data was taken from Refs. [56] (CEA), [57] (Cornell), and [58] (Bebek).

than other uncertainties in our calculation of the vector meson masses.

Second, in the calculation of the form factor, we use the impulse approximation which, in this context, implies neglecting the second and third diagrams in Fig. 3. The consequence of discarding diagrams is the violation of charge conservation or, equivalently, a deviation from $F_{\pi}(0)=1$. As can be seen in the inset in Fig. 5, this effect is of the order of $\sim 1 \%$ only.

As can be also seen from Fig. 5, the results for the pion form factor in the spacelike $Q^{2}>0$ regime are practically independent from the value of the $\eta$ parameter of the model. This is also confirmed by the extracting the charge radius; the corresponding resulting values are $\sqrt{\left\langle r_{\pi}^{2}\right\rangle}=0.685 \mathrm{fm}$ for $\eta=1.5$ and $0.683 \mathrm{fm}$ for $\eta=1.6$. Even more remarkable is the fact that our calculation shows a very good agreement with experimental data, not only for the pion charge radius (the most recent PDG value being $\sqrt{\left\langle r_{\pi}^{2}\right\rangle^{\mathrm{PDG}}}=0.659 \pm 0.004 \mathrm{fm}$ [55]) but in the whole spacelike domain even though, as evident from the above discussion, we aimed at including all physical effects that are important in the timelike regime. An interpretation of this result in view of the dispersion relation (4) provides an indication that the imaginary part in the timelike region is precisely enough reproduced to provide very good results for the spacelike form factor.

We show our results for the pion form factor for timelike $\left(Q^{2}<0\right)$ virtualities in Figs. 6 and 7. As discussed in the previous section, as a consequence of the decay kernels in our truncation, the pion form factor develops a branch cut along the real negative axis, starting from the two-pion threshold $Q^{2}=-4 m_{\pi}^{2}$, induced by the corresponding cut in the quark-photon vertex [33]. Hence, in that region, the (complex) form factor is defined from its analytic continuation as $F\left(Q^{2}+i \epsilon\right)$. In the numerical calculations, we have typically chosen $\epsilon=0.0001 \mathrm{GeV}^{2}$ after verifying that this 


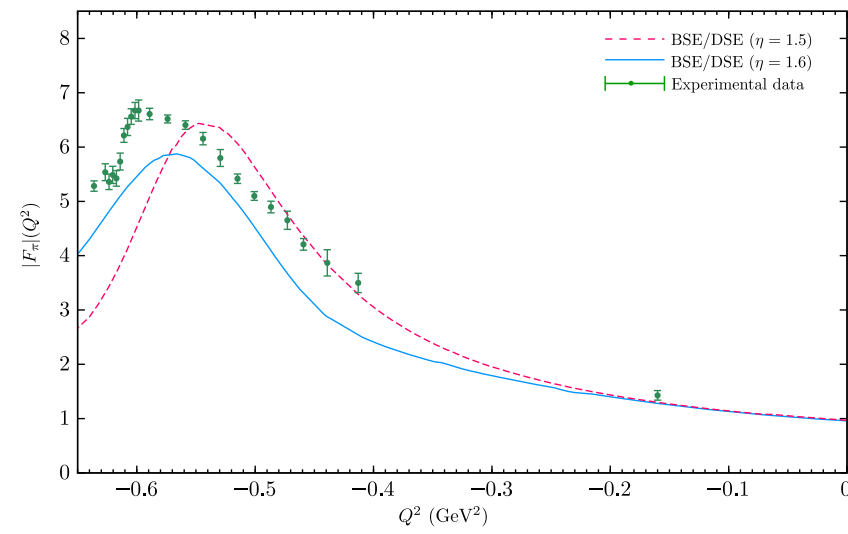

FIG. 6. Absolute value of the pion form factor in the timelike $Q^{2}<0$ domain for the model parameters $\eta=1.5$ and $\eta=1.6$, as described in the text and compared to experimental data [20].

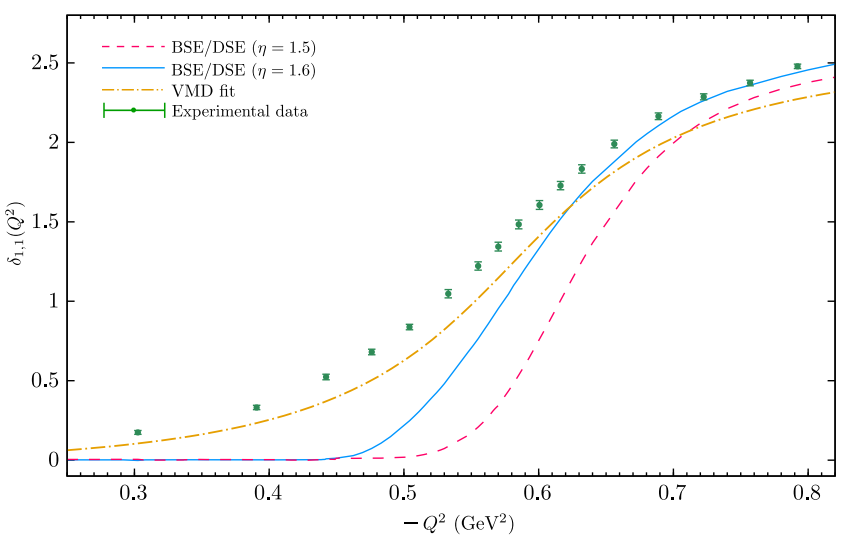

FIG. 7. Phase of the pion form factor in the timelike $Q^{2}<0$ domain for the model parameters $\eta=1.5$ and $\eta=1.6$, as described in the text and compared to experimental data on pion-pion phase shift [62].

value is small enough to not disturb the presented results. In Fig. 6, we present the absolute value for the model parameter $\eta=1.5$ and $\eta=1.6$, with the remaining model parameters kept constant, as discussed above. As a manifestation of the fact that the $\rho$-meson pole in the quarkphoton vertex moves into the complex plane when the decay kernels are included in the calculation, the pion form factor develops a bump on the real and negative $Q^{2}$ axis with an approximately correct height and width. Therefore, our calculation overcomes a major deficiency of the RL truncation, without or even with the pion exchange term, for which the form factor diverges instead at the $Q^{2}$ value corresponding to the $\rho$-meson mass in those truncations; see, e.g., [59-61]. This constitutes already one main result of the here presented investigation.

We note, however, that, contrary to the results for spacelike regime, the position and height of the bump of the form factor depends strongly on the value of the $\eta$
TABLE II. The coefficients of the rational fits to the pion form factor as discussed in the text. All values for dimensionful quantities are given in $\mathrm{GeV}$.

\begin{tabular}{llll}
\hline \hline & $\eta=1.5$ & $\eta=1.6$ & Eq. (8) \\
\hline$a_{1}$ & 0.5587 & 0.4149 & 0.72 \\
$a_{2}$ & 0.8828 & 0.6827 & 1.2 \\
$b_{0}$ & 0.3600 & 0.3600 & 0.36 \\
$b_{1}$ & 1.2307 & 1.2517 & 1.2 \\
$b_{2}$ & 1.0722 & 1.1000 & 1.037 \\
$c_{1}$ & 0.0591 & 0.0997 & 0 \\
$c_{2}$ & 0.1295 & 0.2383 & 0.2308 \\
$d_{0}$ & 0.3600 & 0.3600 & 0.36 \\
$d_{1}$ & 1.1924 & 1.2464 & 1.2 \\
$d_{2}$ & 0.9973 & 1.0916 & 1.037 \\
\hline \hline
\end{tabular}

parameter shape of the form factor. Of course, this reflects the different positions of the $\rho$-meson pole; cf., Table I. Nevertheless, there are features that appear to be independent of $\eta$, most prominently that the height of the bump is underestimated. As expected from the discussion in Sec. II, the form factor behaves smoothly beyond the bump, in contrast to the sharp dip in the experimental data. Even though an unambiguous analysis of the origin of such discrepancies can only result from the inclusion of all relevant physical mechanisms in our calculations, it is evident from the analysis performed in Sec. II that one of the main missing elements, among others, is the $\rho-\omega$ mixing due to isospin breaking, which is completely absent in the present study.

Particularly sensitive to the deficiencies of our truncation is the phase of the form factor, shown in Fig. 7. Even though our data shows the expected behavior near the resonance value, it severely underestimates the experimental data, particularly in the elastic region. This is a manifestation of the absence of some hadronic effects in our approximation scheme, which only includes those stemming from the resonance complex pole in the quark-photon vertex and the $\rho \rightarrow \pi \pi$ induced branch cut. In addition to the isospin breaking effects discussed above, which would be more relevant in the region above the resonance, the impulse approximation used in our calculation of the form factor entails that effects coming from the coupling of the photon to the intermediate hadrons, via its coupling to the exchanged pion or to the quark-pion vertex (see Fig. 3), are missing. It has been shown [63] that considering only impulselike diagrams leads to a very small pion-pion scattering amplitude in the elastic region in the isospin $I=1$ channel. This, due to unitarity, implies a very small value of the imaginary part of the pion form factor in the elastic region (which is, in fact, what we observe), which translates into a very small phase, as seen in Fig. 7 (and as could be inferred from Watson's theorem). 
Last but not least, we are comparing Padé fits, respective rational fits of the order (3.3), for the real part and for the imaginary part of the form factor to the expression (8). Trying first,

$$
\begin{aligned}
\operatorname{Re} F_{\pi}\left(Q^{2}\right)-F_{\pi}(0) & \approx-\frac{a_{0}+a_{1} Q^{2}+a_{2}\left(Q^{2}\right)^{2}+a_{3}\left(Q^{2}\right)^{3}}{b_{0}+b_{1} Q^{2}+b_{2}\left(Q^{2}\right)^{2}+b_{3}\left(Q^{2}\right)^{3}} \\
\operatorname{Im} F_{\pi}\left(Q^{2}\right) & \approx \frac{c_{0}+c_{1} Q^{2}+c_{2}\left(Q^{2}\right)^{2}+c_{3}\left(Q^{2}\right)^{3}}{d_{0}+d_{1} Q^{2}+d_{2}\left(Q^{2}\right)^{2}+d_{3}\left(Q^{2}\right)^{3}},
\end{aligned}
$$

we obtain tiny values for the coefficients $a_{0}, a_{3}, b_{3}, c_{0}, c_{3}$, and $d_{3}$. Note that this confirms the structure expected from the VMD form (8). We repeated the fits for

$$
\begin{aligned}
\operatorname{Re} F_{\pi}\left(Q^{2}\right)-F_{\pi}(0) & \approx-\frac{a_{1} Q^{2}+a_{2}\left(Q^{2}\right)^{2}}{b_{0}+b_{1} Q^{2}+b_{2}\left(Q^{2}\right)^{2}} \\
\operatorname{Im} F_{\pi}\left(Q^{2}\right) & \approx \frac{c_{1} Q^{2}+c_{2}\left(Q^{2}\right)^{2}}{d_{0}+d_{1} Q^{2}+d_{2}\left(Q^{2}\right)^{2}} .
\end{aligned}
$$

The coefficients resulting from these fits, as well as the ones resulting from expression (8), are given in Table II. From this, we conclude that the expression based on the VMD is an astonishingly good representation of our results. Therefore, our investigation makes it plausible that the VMD picture can be derived from QCD. At least, the results of the here presented microscopic approach give a strong hint into this direction.

\section{CONCLUSIONS AND OUTLOOK}

In this work, we have presented an exploratory study of the pion form factor in the DSE and BSE approach. Our focus has been to explore how the interplay between hadron structure (as described by form factors) and the hadron spectrum (as described by resonance masses and widths) can be realized in the microscopic approach presented herein. In particular, we focused on the effect of intermediate pions in the BSE interaction kernel, the inclusion of which is sufficient to describe the $\rho$ meson as a resonance [47]. As elucidated by the detailed analysis in the last section, our calculation represents a verification of the vector meson dominance picture and provides an explanation of how at the quark level, vector meson dominance becomes effective. A more complete calculation than the one presented here might then actually provide a derivation of vector meson dominance from QCD.

Despite the fairly drastic approximations used in this preliminary study, the agreement with experiment is remarkable. On the spacelike side, our calculations agree with experimental data at the quantitative level. For timelike momentum transfers, the agreement is mostly qualitative and consistent with the fact that in our approximation scheme, timelike physics is dominated by the lowest-lying $\rho$-meson resonance only. The absolute value of the calculated form factor features a bump at approximately the correct $Q^{2}$ region as caused by a resonance pole. Our result lacks, however, other features such as those caused by the isospin-breaking $\rho-\omega$ mixing and interference. The phase of the form factor also shows deficiencies caused by the employed impulse approximation. However, the overall qualitatively correct behavior is, nevertheless, very encouraging for future studies on timelike phenomenology with BSE methods as it shows that the necessary computational techniques are getting more and more under control and that within a functional-method-based bound-state approach to $\mathrm{QCD}$, direct calculations in the timelike regime are becoming feasible.

Among the different physical mechanisms absent in our calculation, the most relevant one appears to be isospin breaking by the light quarks' masses and electric charges, and the different phenomena associated with it. The presented exploratory calculation paved the way to include in a bound-state approach formulated in QCD degrees of freedom the effects of isospin violation, and hereby most prominently $\rho-\omega$ mixing, on the timelike pion electromagnetic form factor. Thus, including isospin violation in a BSE approach is the topic of ongoing work. A further related topic is the study of the form factor for the coupling of a photon to three pions. On one hand, this process is of special theoretical interest because the related form factor is at the soft point completely determined by the Abelian chiral anomaly. On the other hand, data of the COMPASS experiment are currently analyzed [64], and therefore, experimental data for this form factor in the timelike region will become available. Combing previous studies in the DSE and BSE approach for the spacelike $\gamma \pi \pi \pi$ form factor [65-69] with the techniques of the here presented calculation will thus enable a respective investigation of this form factor.

Another aspect we want to investigate is how to realize the idea of decay kernels like (A3) and (A4) in a baryon bound state equation. This is a necessary step in order to tackle timelike nucleon form factors in the DSE and BSE approach, which is one of our major goals due to the increased effort and interest from the experimental side in highly precise measurements over a wide kinematical domain of the nucleon form factors.

\section{ACKNOWLEDGMENTS}

We thank Gernot Eichmann and Christian Fischer for a critical reading of the manuscript and helpful discussions. This work was partially supported by the the Austrian Science Fund (FWF) under Project No. P29216-N36. A. S. Miramontes acknowledges CONACyT for financial support. The numerical computations have been performed at the high-performance compute cluster of the University of Graz. 


\section{APPENDIX A: INTERACTION KERNELS}

The BSE kernel representing the exchange of an explicit pionic degrees of freedom as defined in $[43,44]$ reads

$$
\begin{aligned}
K_{r s}^{(t) u t}(q, p ; P)= & \frac{C}{4}\left[\Gamma_{\pi}^{j}\right]_{r u}\left(\frac{p+q-P}{2} ; p-q\right)\left[Z_{2} \gamma^{5}\right]_{t s} D_{\pi}(p-q)+\frac{C}{4}\left[\Gamma_{\pi}^{j}\right]_{r u}\left(\frac{p+q-P}{2} ; q-p\right)\left[Z_{2} \gamma^{5}\right]_{t s} D_{\pi}(p-q) \\
& +\frac{C}{4}\left[Z_{2} \gamma^{5}\right]_{r u}\left[\Gamma_{\pi}^{j}\right]_{t s}\left(\frac{p+q+P}{2} ; p-q\right) D_{\pi}(p-q)+\frac{C}{4}\left[Z_{2} \gamma^{5}\right]_{r u}\left[\Gamma_{\pi}^{j}\right]_{t s}\left(\frac{p+q+P}{2} ; q-p\right) D_{\pi}(p-q),
\end{aligned}
$$

in combination with the following truncation of the quark DSE:

$$
S^{-1}(p)=S^{-1}(p)^{R L}-\frac{3}{2} \int_{q}\left[Z_{2} \gamma_{5} S(q) \Gamma_{\pi}\left(\frac{p+q}{2}, q-p\right)+Z_{2} \gamma_{5} S(q) \Gamma_{\pi}\left(\frac{p+q}{2}, p-q\right)\right] \frac{D_{\pi}(k)}{2},
$$

with $S^{-1}(p)^{R L}$ being the right-hand side of the quark DSE in the RL truncation with the gluon-mediated interaction as described in Sec. III. In Eqs. (A1) and (A2), the pion propagator is taken as $D_{\pi}(k)=\left(k^{2}+m_{\pi}^{2}\right)^{-1}$. The factor $3 / 2$ in Eq. (A2) originates from the flavor traces, and, in the same way, the factor $C$ in (A1) should be obtained. When done in the quark-photon vertex, the flavor factor leads to $C=+3 / 2$. However, such value of $C$ violates the Ax-WTI while preserving the V-WTI. Since the V-WTI is related to charge conservation in electromagnetic form factors, we use $C=+3 / 2$ at the expense of violating the Ax-WTI. Herein, the quark-pion vertex $\Gamma_{\pi}$ is taken to be the pion Bethe-Salpeter amplitude.

The pions in the kernel can also appear in the $\mathrm{s}$ and $\mathrm{u}$ channels [43]. We used here a version of the kernels slightly different to the one in [43] in order to be consistent with the construction for the $t$ channel, where one of the pion vertices is kept bare, and the kernel is then symmetrized. They read

$$
\begin{aligned}
K_{d a}^{(s) h e}(q, p, r ; P)= & \frac{C}{2} D_{\pi}\left(\frac{P+r}{2}\right) D_{\pi}\left(\frac{P-r}{2}\right)\left[\left[Z_{2} \gamma_{5}\right]_{d c} S_{c b}\left(p-\frac{r}{2}\right)\left[Z_{2} \gamma_{5}\right]_{b a}\right. \\
& \times\left[\Gamma_{\pi}^{j}\right]_{h g}\left(q-\frac{P}{4}-\frac{r}{4} ; \frac{r-P}{2}\right) S_{g f}\left(q-\frac{r}{2}\right)\left[\Gamma_{\pi}^{j}\right]_{f e}\left(q+\frac{P}{4}-\frac{r}{4} ;-\frac{P+r}{2}\right) \\
& +\left[\Gamma_{\pi}^{j}\right]_{d c}\left(p+\frac{P}{4}-\frac{r}{4} ; \frac{P+r}{2}\right) S_{c b}\left(p-\frac{r}{2}\right)\left[\Gamma_{\pi}^{j}\right]_{b a}\left(p-\frac{P}{4}-\frac{r}{4} ; \frac{P-r}{2}\right) \\
& \left.\times\left[Z_{2} \gamma_{5}\right]_{h g} S_{g f}\left(q-\frac{r}{2}\right)\left[Z_{2} \gamma_{5}\right]_{f e}\right],
\end{aligned}
$$

and

$$
\begin{aligned}
K_{d a}^{(u) h e}(q, p, r ; P)= & \frac{C}{2} D_{\pi}\left(\frac{P+r}{2}\right) D_{\pi}\left(\frac{P-r}{2}\right)\left[\left[Z_{2} \gamma_{5}\right]_{d c} S_{c b}\left(p+\frac{r}{2}\right)\left[Z_{2} \gamma_{5}\right]_{b a}\right. \\
& \times\left[\Gamma_{\pi}^{j}\right]_{h g}\left(q-\frac{P}{4}-\frac{r}{4} ; \frac{r-P}{2}\right) S_{g f}\left(q-\frac{r}{2}\right)\left[\Gamma_{\pi}^{j}\right]_{f e}\left(q+\frac{P}{4}-\frac{r}{4} ;-\frac{P+r}{2}\right) \\
& +\left[\Gamma_{\pi}^{j}\right]_{d c}\left(p+\frac{P}{4}+\frac{r}{4} ; \frac{P-r}{2}\right) S_{c b}\left(p+\frac{r}{2}\right)\left[\Gamma_{\pi}^{j}\right]_{b a}\left(p-\frac{P}{4}+\frac{r}{4} ; \frac{P+r}{2}\right) \\
& \left.\times\left[Z_{2} \gamma_{5}\right]_{h g} S_{g f}\left(q-\frac{r}{2}\right)\left[Z_{2} \gamma_{5}\right]_{f e}\right],
\end{aligned}
$$




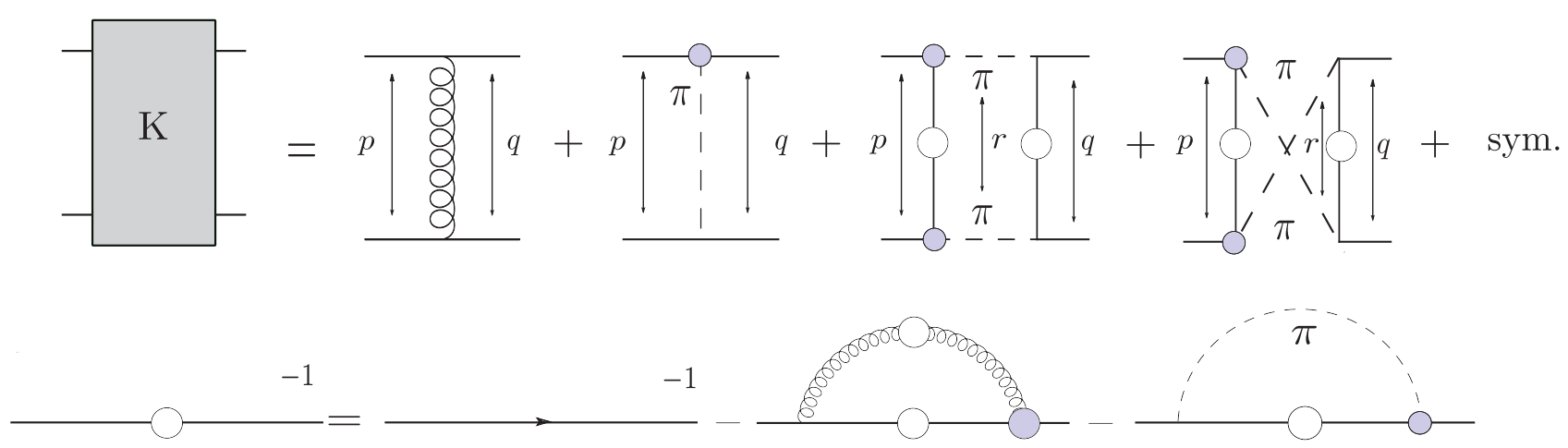

FIG. 8. Truncations used herein for the BSE interaction kernel $K$ (upper diagram) and the quark DSE one (lower diagram). In the upper diagram, the terms on the right-hand side correspond to the rainbow-ladder, pion exchange, and s- and u-channel pion decay contributions to the truncation, respectively. The s- and u-channel pion decay terms do not contribute to the quark DSE.

where now $r$ is an additional integration momentum in the BSE [cf., Eqs. (9) or III]. The resulting truncation of the BSE kernel and the quark DSE is shown in Fig. 8.

The inclusion of the two kernels given in equations (A3) and (A4) generates a highly nontrivial analytic structure of the integrand of the BSE, induced by the intermediate pions going potentially on shell, as well as by singularities in the quark propagators; see Ref. [33] for details, where also the techniques for finding viable contour deformations for performing the numerical integrations in a mathematically correct way are described. ${ }^{10}$

The pion Bethe-Salpeter amplitude possesses four tensor components. Hereby, only one is generically small such that for a precise calculation of pion properties, it would be necessary to take into account three of them, namely the leading pseudoscalar term and two subleading ones related to the axialvector structure. However, in using the above described kernels, three amplitudes for every pion BetheSalpeter amplitude in these expressions is numerically by an order of magnitude more expensive and far beyond the scope of the present study. Having restricted the leading component of the pion amplitude in the kernels (A1) and (A4), one can further exploit that this leading amplitude may be well approximated using the chiral limit value of the quark dressing function $B\left(p^{2}\right)$ and normalize the amplitude by dividing through the pion decay constant $f_{\pi}$,

$$
\Gamma_{\pi}^{i}(p ; P)=\tau^{i} \gamma_{5} \frac{B\left(p^{2}\right)}{f_{\pi}} .
$$

For light quarks, the difference between calculated leading order amplitude and this approximation is at the level of a

\footnotetext{
${ }^{10}$ Further details on applying contour deformations for performing the numerical integrations in the context of DSEs and BSEs can be found in Refs. [46,70-72] and references therein.
}

few percent; see, e.g., [73] and references therein. Therefore, we use this simplified form.

\section{APPENDIX B: POLES OF THE QUARK PROPAGATOR}

In the truncations of the quark DSE used in this paper, the quark propagator features pairs of complex conjugate poles in the complex plane (see, e.g., [74]). In order to facilitate the use of the quark propagators and easily identify the analytic structures generated by those poles in the form factor and vertex calculations, it is useful to parametrize the quark propagator simply as a sum of poles (see, e.g., [75]),

$$
\begin{aligned}
S(p) & =-i \not p \sigma_{v}\left(p^{2}\right)+\sigma_{s}\left(p^{2}\right), \\
\sigma_{v}\left(p^{2}\right) & =\sum_{i}^{n}\left[\frac{\alpha_{i}}{p^{2}+m_{i}}+\frac{\alpha_{i}^{*}}{p^{2}+m_{i}^{*}}\right], \\
\sigma_{s}\left(p^{2}\right) & =\sum_{i}^{n}\left[\frac{\beta_{i}}{p^{2}+m_{i}}+\frac{\beta_{i}^{*}}{p^{2}+m_{i}^{*}}\right],
\end{aligned}
$$

where the parameters $m_{i}, \alpha_{i}, \beta_{i}$ can be obtained by fitting the corresponding quark DSE solution along the $p^{2}$ real axis or, alternatively, on a parabola in the complex plane that does not enclose the poles.

In the numerical solution of the quark DSE in the complex plane, we tested fits with one real and one pair of complex conjugated poles, with two pairs of complex conjugated poles and with three pairs of complex conjugated poles. Based on this tests, we concluded that $n=2$ pairs of complex conjugated poles provided a precise fit, and as the use of three pairs of poles did not provide any further improvement, the reported calculations have been performed based on fits with two pairs of poles. 
[1] W. Melnitchouk, R. Ent, and C. Keppel, Phys. Rep. 406, 127 (2005).

[2] I. C. Cloet and C. D. Roberts, Prog. Part. Nucl. Phys. 77, 1 (2014).

[3] G. Eichmann, H. Sanchis-Alepuz, R. Williams, R. Alkofer, and C. S. Fischer, Prog. Part. Nucl. Phys. 91, 1 (2016).

[4] M. Q. Huber, Phys. Rep. 879, 1 (2020).

[5] H. Sanchis-Alepuz and R. Williams, Comput. Phys. Commun. 232, 1 (2018).

[6] M. Q. Huber, C. S. Fischer, and H. Sanchis-Alepuz, Eur. Phys. J. C 80, 1077 (2020).

[7] K. Langfeld, R. Alkofer, and P. A. Amundsen, Z. Phys. C 42, 159 (1989).

[8] C. D. Roberts, Nucl. Phys. A605, 475 (1996).

[9] P. Maris and P. C. Tandy, Phys. Rev. C 62, 055204 (2000).

[10] L. Chang, I. C. Cloët, C. D. Roberts, S. M. Schmidt, and P. C. Tandy, Phys. Rev. Lett. 111, 141802 (2013).

[11] E. P. Biernat, F. Gross, T. Peña, and A. Stadler, Phys. Rev. D 89, 016006 (2014).

[12] D. Nicmorus, G. Eichmann, and R. Alkofer, Phys. Rev. D 82, 114017 (2010).

[13] G. Eichmann, Phys. Rev. D 84, 014014 (2011).

[14] G. Eichmann and C. S. Fischer, Eur. Phys. J. A 48, 9 (2012).

[15] H. Sanchis-Alepuz, R. Williams, and R. Alkofer, Phys. Rev. D 87, 096015 (2013).

[16] H. Sanchis-Alepuz, R. Alkofer, and C. S. Fischer, Eur. Phys. J. A 54, 41 (2018).

[17] G. Barucca et al. (PANDA Collaboration), arXiv: 2101.11877.

[18] B. Ananthanarayan, I. Caprini, and D. Das, Phys. Rev. D 102, 096003 (2020).

[19] J. H. Kim and L. Resnick, Phys. Rev. D 21, 1330 (1980).

[20] R. R. Akhmetshin et al. (CMD-2 Collaboration), Phys. Lett. B 648, 28 (2007).

[21] D. Brömmel et al. (QCDSF/UKQCD Collaborations), Eur. Phys. J. C 51, 335 (2007).

[22] R. Frezzotti, V. Lubicz, and S. Simula (ETM Collaboration), Phys. Rev. D 79, 074506 (2009).

[23] P. A. Boyle, J. M. Flynn, A. Juttner, C. Kelly, H. P. de Lima, C. M. Maynard, C. T. Sachrajda, and J. M. Zanotti, J. High Energy Phys. 07 (2008) 112.

[24] S. Aoki et al. (JLQCD and TWQCD Collaborations), Phys. Rev. D 80, 034508 (2009).

[25] O. H. Nguyen, K. I. Ishikawa, A. Ukawa, and N. Ukita, J. High Energy Phys. 04 (2011) 122.

[26] B. B. Brandt, A. Jüttner, and H. Wittig, J. High Energy Phys. 11 (2013) 034.

[27] J. Koponen, F. Bursa, C. Davies, G. Donald, and R. Dowdall, Proc. Sci., LATTICE2013 (2014) 282.

[28] H. B. Meyer, Phys. Rev. Lett. 107, 072002 (2011).

[29] X. Feng, S. Aoki, S. Hashimoto, and T. Kaneko, Phys. Rev. D 91, 054504 (2015).

[30] J. Bulava, B. Hörz, B. Fahy, K. J. Juge, C. Morningstar, and C. H. Wong, Proc. Sci., LATTICE2015 (2016) 069.

[31] F. Erben, J. R. Green, D. Mohler, and H. Wittig, Phys. Rev. D 101, 054504 (2020).

[32] J. P. B. C. de Melo, T. Frederico, E. Pace, and G. Salme, Phys. Rev. D 73, 074013 (2006).

[33] Á. S. Miramontes and H. Sanchis-Alepuz, Eur. Phys. J. A 55, 170 (2019).
[34] H. B. O'Connell, B. C. Pearce, A. W. Thomas, and A. G. Williams, Prog. Part. Nucl. Phys. 39, 201 (1997).

[35] F. Jegerlehner and R. Szafron, Eur. Phys. J. C 71, 1632 (2011).

[36] S. Schael et al. (ALEPH Collaboration), Phys. Rep. 421, 191 (2005).

[37] M. Davier, A. Hocker, and Z. Zhang, Rev. Mod. Phys. 78, 1043 (2006).

[38] H. Y. Chen and H. Q. Zhou, Phys. Rev. D 98, 054003 (2018).

[39] I. J. R. Aitchison and A. J. G. Hey, Gauge Theories in Particle Physics: A Practical Introduction. Vol. 1: From Relativistic Quantum Mechanics to QED, 3rd ed. (IoP Publishing, Bristol and Philadelphia, 2003).

[40] D. Melikhov, O. Nachtmann, and T. Paulus, arXiv:hep-ph/ 0209151.

[41] P. Maris and C. D. Roberts, Phys. Rev. C 56, 3369 (1997).

[42] P. Maris and P. C. Tandy, Phys. Rev. C 60, 055214 (1999).

[43] C. S. Fischer, D. Nickel, and J. Wambach, Phys. Rev. D 76, 094009 (2007).

[44] C. S. Fischer, D. Nickel, and R. Williams, Eur. Phys. J. C 60, 47 (2009).

[45] R. Alkofer, A. Bender, and C. D. Roberts, Int. J. Mod. Phys. A 10, 3319 (1995).

[46] N. Santowsky, G. Eichmann, C. S. Fischer, P. C. Wallbott, and R. Williams, Phys. Rev. D 102, 056014 (2020).

[47] R. Williams, Phys. Lett. B 798, 134943 (2019).

[48] D. Jarecke, P. Maris, and P. C. Tandy, Phys. Rev. C 67, 035202 (2003).

[49] V. Mader, G. Eichmann, M. Blank, and A. Krassnigg, Phys. Rev. D 84, 034012 (2011).

[50] H. Haberzettl, Phys. Rev. C 56, 2041 (1997).

[51] A. N. Kvinikhidze and B. Blankleider, Phys. Rev. C 60, 044003 (1999).

[52] A. N. Kvinikhidze and B. Blankleider, Phys. Rev. C 60, 044004 (1999).

[53] M. Oettel, M. Pichowsky, and L. von Smekal, Eur. Phys. J. A 8, 251 (2000).

[54] M. Oettel, R. Alkofer, and L. von Smekal, Eur. Phys. J. A 8, 553 (2000).

[55] P. A. Zyla et al. (Particle Data Group), Prog. Theor. Exp. Phys. 2020, 083C01 (2020).

[56] C. N. Brown, C. R. Canizares, W. E. Cooper, A. M. Eisner, G. J. Feldmann, C. A. Lichtenstein, L. Litt, W. Loceretz, V. B. Montana, and F. M. Pipkin, Phys. Rev. D 8, 92 (1973).

[57] C. J. Bebek, C. N. Brown, M. Herzlinger, S. D. Holmes, C. A. Lichtenstein, F. M. Pipkin, L. K. Sisterson, D. Andrews, K. Berkelman, and D. G. Cassel et al. Phys. Rev. D 9, 1229 (1974).

[58] C. J. Bebek, C. N. Brown, M. Herzlinger, S. D. Holmes, C. A. Lichtenstein, F. M. Pipkin, S. Raither, and L. K. Sisterson, Phys. Rev. D 13, 25 (1976).

[59] P. Maris and P. C. Tandy, Phys. Rev. C 61, 045202 (2000); Nucl. Phys. B, Proc. Suppl. 161, 136 (2006).

[60] A. Krassnigg and P. Maris, J. Phys. Conf. Ser. 9, 153 (2005).

[61] G. Eichmann, C. S. Fischer, E. Weil, and R. Williams, Phys. Lett. B 797, 134855 (2019); 799, 135029(E) (2019).

[62] S. D. Protopopescu, M. Alston-Garnjost, A. Barbaro-Galtieri, S. M. Flatte, J. H. Friedman, T. A. Lasinski, G. R. Lynch, M. S. Rabin, and F. T. Solmitz, Phys. Rev. D 7, 1279 (1973). 
[63] S. R. Cotanch and P. Maris, Phys. Rev. D 66, 116010 (2002).

[64] J. Friedrich and D. Steffen (private communication).

[65] R. Alkofer and C. D. Roberts, Phys. Lett. B 369, 101 (1996).

[66] B. Bistrovic and D. Klabucar, Phys. Lett. B 478, 127 (2000).

[67] S. R. Cotanch and P. Maris, Phys. Rev. D 68, 036006 (2003).

[68] S. Benic and D. Klabucar, Phys. Rev. D 85, 034042 (2012).

[69] G. Eichmann and C. S. Fischer, Phys. Rev. D 85, 034015 (2012).
[70] A. Windisch, M. Q. Huber, and R. Alkofer, Acta Phys. Pol. B Proc. Suppl. 6, 887 (2013).

[71] A. Windisch, Features of strong quark correlations at vanishing and non-vanishing density, Ph.D. thesis, University of Graz, 2014.

[72] G. Eichmann, P. Duarte, M. T. Peña, and A. Stadler, Phys. Rev. D 100, 094001 (2019).

[73] C. S. Fischer and R. Williams, Phys. Rev. D 78, 074006 (2008).

[74] A. Windisch, Phys. Rev. C 95, 045204 (2017).

[75] B. El-Bennich, G. Krein, E. Rojas, and F. E. Serna, Few Body Syst. 57, 955 (2016). 